\title{
Bodily Awareness and Its Subject
}

\author{
Naoyuki SHIono*
}

\section{Introduction}

This paper will examine the relevance of a particular form of awareness which we have of our own body to the problem of the self. The phenomena we are going to look at include proprioceptive awareness and sensations which have specific bodily location. Proprioceptive awareness is the form of awareness through which we know the relative position of our limbs without seeing or touching them. For example, one can know that one's legs are crossed without observing them visually or tactually from outside. Sensations which have bodily location, such as pain and itch, share the same characteristics as proprioceptive awareness in that they provide us with direct, unmediated knowledge of the condition of our specific body parts. Kinaesthetic sensations, temperature and pressure of our body parts may also be incorporated in our scope. We will not, however, be concerned with subtle differences between those modes of awareness, and will treat them as one particular form of awareness of our own body and call it 'bodily awareness'.

On the other hand, the 'self', or the 'subject', is to be understood as the subject of experience, such as thought, perception, sensation, emotion and so on. Thus, our inquiry is into whether bodily awareness reveals anything about the subject of those various forms of experience. Bodily awareness is, of course, one form of experience, and, on the face of it, it has a subject and an object. Its object is, needless to say, the body and its conditions. But what is its subject? Does an investigation into bodily awareness tell us anything of interest about the nature of the self? This is the question this paper undertakes to explore. Recently, some philosophers, including Evans (1982: 220-222), Brewer (1995), Cassam (1995; 1997) and Bermúdez (1998: 131-162), have claimed that considerations on bodily awareness will persuade us to accept that the subject is a corporeal, bodily entity, which has a spatial location and other physical properties. They argue that the subject of experience is the object of bodily awareness, in other words, that in bodily awareness one is presented to oneself as a subject and also as a corporeal entity. This proposal is what we are going to examine, and our conclusion will be that, contrary to their view, reflections on bodily awareness do not have any such consequence.

It is important that in our starting point we should not commit ourselves to any specific conception of the subject. The minimal assumption with which we begin is

\footnotetext{
* The University of Tokyo
} 
that there are such entities as subjects of experience, and that the first-personal pronoun ' $I$ ' used in judgements in which one ascribes experiences to oneself refers to oneself. With this assumption, we will examine various attempts which purport to show that bodily awareness reveals the physical nature of the self. However, one may think that even this minimal assumption is not warranted, if one agrees with Hume that it is misguided to think that experience has a subject as well as an object. In fact, this Humean position will eventually be left as a possibility, because the conclusion we expect to draw from the above assumption is a negative one that considerations on bodily awareness do not establish anything about the subject of experience.

\section{How We Locate Bodily Awareness}

Philosophers sympathetic to a broadly Cartesian line of thought may reason that experience must be qualitatively given, and it is only contingently related to the external physical world. This is because, according to this conception, one cannot be mistaken about what kind of qualitative experience one is having, while one can go wrong as to the external world which the experience is about. This familiar thought may be flawed in many ways, but our interest here lies in the point that bodily awareness seems to give us a manifest counterexample to this conception of experience. Suppose I have a pain in my left leg. If the Cartesian picture is correct, I do not directly locate the pain in my leg. Rather, apart from the painful quality, the experience has another qualitative feature, call it left-legginess, from which I inductively infer that the pain is there. When I feel an itch in my left leg, left-legginess should again be present, together with the itchy feeling. When I have a pain in my back, the pain must be accompanied by another qualitative feature of experience that tells me that the pain is instantiated in my back. In this way, the Cartesian picture requires that there should be a whole range of distinctive qualitative character of experience which will be only empirically correlated with each part of our body.

However, as Anscombe (1962) has first pointed out with respect to proprioceptive awareness, and as O'Shaughnessy (1980), among others, has argued, it is wrong to believe in such a qualitative feature of experience from which we empirically infer the location of a sensation. Rather, we locate pain or itch directly to our body parts. This is not to say that we cannot be mistaken about where we have a sensation. We do make that kind of mistake. But, if we accept the broadly Cartesian conception, it will be as if we have to go through an inference to find out in which body part a sensation is present, because what we are immediately aware of is supposed to be purely qualitative. And this idea seems absolutely wrong.

Now, it is not obvious what bearing this objection against Cartesianism has on 
our concern, on our inquiry about the subject of experience. Certainly, it shows that we should think of the subject as having some special direct access to a particular object, its body. This might furthermore give us some motivation to believe that the subject must have the same spatial location as the body. However, it achieves no more than this. After all, Anscombe's observation characterises the body as nothing other than an object of awareness, and it does not commit us to any specific view about its subject. We are, therefore, not yet in a position to draw the further conclusion that the subject must be identical with the body. It requires much more argument to show that the object of bodily awareness is the subject of that very awareness.

Brewer (1995: 300-303) has tried to establish that conclusion by developing Anscombe's idea. According to him, when I have a pain in my leg, we should describe the body part itself as the subject of the psychological property. He thinks that, in this case, what I am aware of is that the leg is in the state of being in pain, and being in pain is a proper psychological state. In this way, psychological states are instantiated in the body part, and this he argues commits us to describing the body itself as the subject of psychological properties. Thus Brewer's thought is that the significance of bodily awareness lies in offering a relatively straightforward proof of the anti-Cartesian conclusion.

This proposal, however, seems neither attractive nor persuasive. Surely it is true that I locate pain directly in my leg. But, instead of accepting Brewer's description, we may try to explain this by claiming that what I attribute to the leg is nothing more than the physical property of being damaged, and this does not involve attributing the psychological state itself to the body part. Indeed, such a description should be favoured because it does not apparently make sense to say that the leg has an experience. The psychological property should rather be attributed to me, to the subject, and we are not yet offered any reason to think that $I$, the subject, must be corporeal. If we assimilate bodily awareness to perception, the weakness of Brewer's argument becomes evident. I perceive that my leg is damaged, and this perception makes me feel painful and unpleasant. Just in the same way, I may see a beautiful landscape and I may feel delighted. Clearly, neither of them establishes the physical nature of the self.

\section{Immunity to Error through Misidentification}

It seems that we need a more sophisticated reasoning than Anscombe's and Brewer's if we want to support the thesis that the subject of experience is a corporeal entity. Discussions on the first-personal pronoun employed in psychological judgements have often centred upon the phenomenon of immunity to error through misidentification relative to the subject, which has first been identified by Wittgen- 
stein in the Blue Book (66-7) and developed, among others, by Shoemaker (1968). We will now move on to this issue. First, we will introduce the notion, and then examine how the phenomenon is exhibited in the case of bodily awareness. Finally, we will see whether it can play any illuminating role in the understanding of the self.

To take an example, to say that the judgement ' $\mathrm{I}$ am thinking about politics' is immune to error through misidentification relative to the subject of the state of affairs being judged is to say the following. It cannot be the case that the judgement is made on the basis of the speaker's belief, gained in the relevant way, namely by reflection, that a particular person is thinking about politics, but the judgement is mistaken for the reason that the speaker wrongly believes that the person thinking about politics is himself while in fact it is someone else. This is not to say that the judgement is free from any kind of mistake. It is to say that it cannot be in error in the particular way specified above. That is, it cannot be mistaken about the identity of ' $I$ '. Such a mistake is not simply highly unlikely but does not make sense. This case is contrasted with those uses of the first-personal pronoun where it is possible to make that sort of error. For example, if someone judges ' $I$ weigh 70 $\mathrm{kg}$ ' on the basis of reading a scale, it is possible that the person on the scale is, contrary to his belief, not himself but someone else, and consequently the judgement is mistaken with respect to ' $T$ '. From this, Wittgenstein has observed that the kind of immunity to error he has identified is a unique characteristic of psychological self-ascription, that is, ascription to oneself of thought, perception, sensation, emotion and so on.

However, it is not true that immunity to error through misidentification relative to the subject is a feature which can be discerned exclusively in psychological selfascription. As Evans (1982: 180) has pointed out, the possibility of misidentification arises only when the judgement contains an identification component. That is, misidentification can occur only when a judgement of the form ' $a$ is $F$ ' is based on corresponding judgements of the form ' $a=b$ ' and ' $b$ is $F$ '. Therefore, when someone judges 'Mary is tall' on the basis of his observation that the person in front of him is tall and of his belief that she is Mary, there is a possibility of misidentification because he may have gone wrong in believing that she is Mary. In our previous example, 'I weigh $70 \mathrm{~kg}$ ' can be mistaken because the identification component 'I am the person on the scale' or 'My body is the body on the scale' can be mistaken.

On the other hand, if someone judges 'She is tall', solely on the basis of his direct observation of the person, to whom he refers by using 'she' as a demonstrative expression, then it seems that he cannot be in error about the identity of 'she', because there is no identification component in Evans's sense involved in this judgement. The judgement is not a consequence drawn from a pair of his beliefs 
that she is some particular person and that that particular person is tall. Evans has thus observed that immunity to error through misidentification is a characteristic also of demonstrative identification. Indeed, we may go further than Evans and claim that misidentification does not immediately seem possible in our ordinary use of proper names. True, if one uses the proper name 'Mary' to refer to the person whom one directly observes and believes to be Mary, then there is a possibility of misidentification. But, if one for example makes an assertion that Napoleon was defeated in Russia by reading a history book, it is not at all clear how one can make a misidentification relative to the subject of the state of affairs being asserted, 'Napoleon'. It is not easy to see what kind of identification component can be detected here. To say the least, that would need a very complicated theory of proper names in the background.

\section{Immunity to Error Relative to the Owner}

Now let us return to our main concern. What we are going to examine is the claim that immunity to error through misidentification can be observed in our judgements based on bodily awareness in the following way. When someone judges 'My right arm is bent', it cannot be the case that the judgement is made on the basis of his belief, gained in the relevant way, in this case by proprioceptive awareness, that a particular limb is bent, but the judgement is mistaken for the reason that he wrongly believes that the limb being bent is his own while in fact it is not. Indeed, on the face of it, such a mistake appears to be impossible, simply because there seems something incoherent about its possibility.

How is this observation supposed to support the idea that the self is a corporeal entity? This is not a straightforward matter. It could support that idea if immunity to error through misidentification were a peculiarity of reference to oneself as a subject of experience. In that case, it could seem plausible to argue that, since reference to one's body through bodily awareness also exhibits the immunity to error, the body of which one is thus aware must be the subject. However, this strategy does not work, because, as we have seen, the immunity is a widespread phenomenon exhibited by demonstratives and perhaps even in many cases of our use of proper names. It may remain true that, as far as first-personal pronoun is concerned, the immunity can be used as a criterion to distinguish psychological selfascription from other kinds of self-ascription. But we must recognise that, unless we scrutinise the sort of judgement in question in more detail, we have not yet found any plausible support for the idea.

When it is claimed that, when someone judges 'My legs are crossed' on the basis of proprioceptive awareness, the judgement exhibits immunity to error through misidentification, this is not, or at any rate should not be, the claim that the 
judgement cannot be in error about the identity of the subject of the state of affairs being judged, 'my legs'. It can easily be imagined that someone wrongly believes that his third finger and his middle finger are crossed, while the fact is that his middle finger and his index finger are crossed, and the mistaken belief is formed on the basis of his proprioceptive awareness of this state of affairs. Then, nothing seems to exclude the possibility that he wrongly believes that his legs are crossed, while the fact is that his arms are crossed, and the mistaken belief is formed owing to his awareness of this state of affairs. Although the latter may hardly happen, the difference between these two cases must be a difference of degree. Thus, the judgement in question cannot be immune to error relative to 'my legs'.

The immunity to error through misidentification to be observed, if it exists at all, should rather be relative to the possessive pronoun 'my'. That is to say, it should be about the identity of ' $I$ ' to whom the body part in question belongs. When someone judges 'My legs are crossed', it seems that it cannot be the case that the judgement is made on the basis of his belief, gained by proprioceptive awareness, that particular limbs are crossed, but it is mistaken for the reason that he wrongly believes that the limbs being crossed are his own while in fact they are not. The judgement may be mistaken in various other ways. Perhaps his legs are not crossed, or perhaps it is not his legs but his arms that are crossed. The claim is that it cannot be mistaken in the way specified. In general, the immunity being put forward is this. One cannot be mistaken in thinking of some object to be one's own body part, as far as the object is presented to one through bodily awareness. We shall shortly examine whether this immunity really obtains. But, if it does obtain, the observation may secure the physical nature of the self in the following way.

The use of a possessive pronoun, as in 'my legs', indicates that there is apparently an ownership relation to be found here. Thus we may label the phenomenon 'immunity to error relative to the owner'. But what is the thing owned, and what is its owner? The thing being owned is, of course, the body or the body parts. On the other hand, the entity which is supposed to be the 'owner' of the body must be the subject. In a judgement 'I have a pain in my leg', ' $\mathrm{I}$ ' and 'my' would refer to the same entity. ' $I$ ' refers to the subject, given the definition that it is the entity to which experiences are attributed. Then the possessive pronoun should also refer to the subject, which means that it is the owner of the body. This so far does not commit us to any specific notion of the subject. We are simply operating with the minimal assumption, which we have made at the outset, that there are such entities as subjects of experience. Now it remains to see what exactly this ownership relation between the subject and the body amounts to, and what consequence it has on our enquiry into the self.

It was claimed above that the subject cannot make a mistake whether any body part belongs to it or not, as far as the body part is presented to it through bodily 
awareness. In other words, there cannot be an error about the identity of the 'owner' of the body part. This is what is indicated by the immunity to error relative to the owner. Then, there must be a very strong connexion between the owner, which is the subject, and its body parts. It is different from that which holds, for example, between a house and its owner, because in this case the owner can make a mistake of thinking of a particular house as his own while in fact it is not. The relation which obtains between the subject and the body appears peculiarly strong, which does not seem to obtain in other cases in which we talk about ownership. What makes a house belong to someone is such facts as he lives in it or he has purchased it. But nothing of this kind is true of a subject and its body parts.

Now if we take it that the subject is itself a corporeal entity, that it is the living human body which comprises the body parts in question, then this fact seems to receive a reasonable explanation. The apparent ownership by the subject of the body parts turns out, on this view, to be a whole-part relation between the body and its parts. This is certainly stronger than the relation between a house and its owner. Moreover, neither is it an ordinary whole-part relation. Living human body is an organism, and its parts are linked with each other in a sophisticated manner to form a highly integrated unity. This integration is realised, among other things, by the human nervous system, and what is important is the fact that this is exactly what makes bodily awareness available to us. It is this organic integration sustained by the nervous system that physically underlies the capacity of the subject to produce judgements based on bodily awareness. Although the subject can be aware of its own body through various modes of awareness, for example by seeing it, bodily awareness is unique among them in exploiting the above function of a living human body. This being what gives bodily awareness the privilege of being immune to error relative to the owner, the immunity seems to support the thesis that the subject is identical with the body of which it is aware. In other words, bodily awareness is, on this explanation, the unique form of awareness through which one is presented to oneself as a subject and also as a corporeal entity.

It is not entirely clear how conclusive the above argument is. In fact, immunity to error relative to the owner is itself a widespread phenomenon not apparently confined to bodily awareness. For example, if someone says ' $\mathrm{My}$ legacy should be divided equally between my children', it does not seem possible that he could make the mistake of thinking about someone else's legacy, and in this case there is nothing extraordinary about the relation between his legacy and himself. However, it seems fair to say that, if bodily awareness is to have any implication on the physical nature of the self, it must be something along the above line of thought, which centres upon the notion of immunity to error relative to the owner, which will approve the implication. And this suffices for our present purposes, as we are going to see in detail whether judgements made on the basis of bodily awareness do exhibit such 
immunity.

\section{Being Aware of Someone Else's Body}

We now turn to examine if there really is immunity to error through misidentification relative to the owner in judgements based on bodily awareness. If this can be revealed not to be the case, it means that the current project has failed to get off the ground. What we are going to consider is the possibility of having proprioceptive awareness of, or feeling sensation in, someone else's body. This, of course, does not usually happen, but apparently there seems nothing inconsistent in this piece of imagination. And if this can be shown to be a genuine possibility, then the argument being offered for the identity of the subject with a corporeal entity will no longer be tenable, because the purported immunity has proven not to exist.

Let us suppose that we have here two living human bodies, A and B. Names ' $A$ ' and ' $B$ ' refer to those corporeal entities. And let us also suppose that $I$ am $A$. Our situation is that I am A in the ordinary sense of the word, that is, A is my body. Under normal circumstances, if I make a judgement through bodily awareness, it does not happen that the body part judged to be thus and so is not part of $\mathrm{A}$. Whenever I make such judgements as 'My legs are crossed', they are about A, although it may happen that A's legs are not actually crossed and I am mistaken in believing that they are. Now if, further, we can somehow demonstrate that under no possible circumstance my judgement based on bodily awareness can fail to be about my own body, then we can claim that the judgement does exhibit immunity to error relative to the owner, and this result will encourage the idea that I am identical with a corporeal entity in the way we have discussed.

There is one issue about which we must be clear at this point. We gave our initial situation by the description ' $I$ am A in the ordinary sense of the word'. What does this mean? What is the ' $\mathrm{I}$ ' here supposed to be referring to ? Is this an identity statement? Or are we expecting to find some deeper analysis of what is meant by ' $\mathrm{I}$ am A'? These questions should be answered in the following way. This use of ' $I$ ' is certainly intended to refer to the subject, which is the 'owner' of the body A. But we do not yet have any analysis of the statement ' $\mathrm{I}$ am A'. That is exactly what we are looking for. If it turns out to be an identity statement, then we have what we wanted, namely, a demonstration that the subject is a corporeal entity. But otherwise, we may have to accept a different conclusion. And it is in the hope of finding a plausible analysis that we examine the abnormal case which we introduce now.

We are to consider the possibility that $I$ am aware of part of $B$, in addition to A, through bodily awareness. For example, let us suppose that I can tell the posture of B's right arm as if through proprioceptive awareness, that is, without 
visually or tactually perceiving it, and I can also feel sensation in it. Apparently there is no difficulty in imagining this. Some more detail, however, needs to be added. First, let us suppose that my judgements as to the condition of B's arm made on the basis of this apparent bodily awareness are almost always correct, so that it is not reasonable to say that they are true simply by chance. Second, there is no reason to reject this imagined case on the ground that it is physically impossible. We may suppose that a pair of radio transmitters are embedded in A's brain and B's right arm, so that information can constantly be exchanged between the two. Third, however, creating an explanation of this science fiction style does not seem essential for our purposes. We may leave open the question of what physically underlies my awareness of B's arm and its reliability. We can think of a situation in which we simply do not know how it is physically realised. In this circumstance one might perhaps think that there must be a yet undiscovered physical mechanism at work, but one need not think so.

It is part of our imagined situation that I may make such mistakes as thinking that A's arm is bent while in fact B's arm is, and the mistaken belief is formed owing to my awareness of the latter. We have seen a case before where I wrongly believe that my third finger and my middle finger are crossed, while in fact my middle finger and my index finger are crossed. The case at hand is exactly parallel to this, and we have no ground for excluding its possibility. It may also happen that $I$ am aware only that some arm is bent, and wonder whether it is A's or B's, just as I may be aware that either my middle finger or my third finger is bent, but am not sure which. It is these cases that seem to threaten the immunity to error relative to the owner, because, on the face of it, I have bodily awareness of someone else's body part and am mistaken in thinking that it is mine or it may be mine. Now, how are we to deal with this abnormal circumstance? There are three strategies available to us at this point, and we will examine each of them one by one. As we shall see, however, none of them will eventually support the thesis that the self is a corporeal entity.

\section{The First Strategy}

The first strategy declares that in the imagined situation B's arm is part of my body. I have genuine bodily awareness of it, and that makes it part of my body. My body consists of, presumably, the whole of body A and B's right arm. If this response is tenable, immunity to error through misidentification relative to the owner will be secured, because, after all, anything of which I am aware through bodily awareness necessarily belongs to me, even if it is not part of $A$. It will be a sufficient condition, if not a necessary condition, of something's being my body part that I have bodily awareness of it. Now, this strategy has considerable 
difficulties. But we will start by dealing with what seem to be rather superficial and surmountable objections.

First, it may be replied that it is counterintuitive to claim that B's arm, in spite of being remote from the rest of my body, is part of it. My body parts must be physically united, and anything which fails to satisfy this constraint cannot be part of my body. This objection, though a sensible one, is not as strong as to immediately discredit the current approach altogether. If it is only our intuition that supports the above constraint, then we should remind ourselves that we are imagining a case which in fact has never obtained, and this implies that we should not rely so heavily on our intuition here. The current proposal is consistent with our ordinary linguistic practise under normal circumstances, and this seems to provide us some reason at least to pursue seriously the prospect of accepting the proposal, and taking it as a norm which determines what we should say in an abnormal situation, notwithstanding our intuition.

Second, should we say that B's arm is part of my body, even if I cannot move it? This objection is not threatening either. There are many parts of my body, like internal organs, of which I have neither control nor awareness, but which clearly belong to me. There are some other parts, such as skin, which I cannot control but of whose condition I am aware, and which definitely belong to me. Therefore no argument can make it a necessary condition that I should be able to control it for it to count as part of my body. Actually, it seems that having bodily awareness is a necessary condition of being able to control. It is difficult to conceive of any body part of whose relative position I am not aware through proprioceptive awareness, but which nevertheless I can move. This may suggest that bodily awareness is more basic than control, and we are well advised to concentrate on the former. But we may note that having control has to do with will and action, which may play a significant role in the problem of the self. This, however, is not in the scope of this paper.

Thus, this strategy does not seem to involve any inconsistency. However, although it secures the immunity to error relative to the owner, it is not going to give any convincing support for the desired conclusion that the subject of experience is a corporeal entity, for the following reason. The position is, as we have seen, that the sufficient condition for something to be part of my body is that I am aware of it through bodily awareness. Let us remind ourselves here that our enquiry has been on the clarification of the relation between the subject, which is the owner of the body, and the body owned by it. Now we have an answer to this enquiry. The relation in question is, basically, 'being bodily aware of'. Bodily awareness is constitutive of the relation between a subject and its body. In other words, setting aside details as to how to incorporate those parts like internal organs into the picture, the question 'Does this body part belong to me ?' necessarily invokes the question 
'Am I, as a subject of experience, aware of it?' Then, not only of B's arm but also of $A$, we should claim that it belongs to me because $I$, as a subject, am aware of it through bodily awareness. That body $\mathrm{A}$ stands in this particular relation to me makes it the case that $A$ is my body. It follows that even under normal circumstances where I do not have any awareness other than of $A$, we should think that it is my awareness of $\mathbf{A}$ which constitutes the fact that $\mathbf{A}$ is my body, that is, I am $\mathbf{A}$ 'in the ordinary sense of the word'.

Now, in order to arrive at the desired conclusion, what has to be demonstrated is that the relation 'being bodily aware of', or a sufficient refinement of it that would accommodate those parts like internal organs of which I am not aware, turns out to be an identity relation. Only then, we will be able to say that $I$, the subject, am identical with the totality of A and B's arm in the imagined situation. And equally, only that demonstration will show that, under normal circumstances, I am identical with the living human body $\mathrm{A}$. However, nothing so far in our argument points in this direction. On the contrary, the concept of the subject was presupposed in the specification of which body part, and which body, belongs to me. That is, only when both I, the subject, and the relation 'being bodily aware of' are properly in place will we be able to start looking for my body, because what counts as my body is, basically, whatever I am aware of through bodily awareness. It is fair to say at this point that we are not offered any reason to identify the subject with the body of which it is aware. The latter can be specified only in terms of the former, and this counts against, or at least does not support, identifying the two.

We previously did hold that immunity to error relative to the owner, if it obtains with respect to our judgements based on bodily awareness, may confirm the idea that the subject is a corporeal entity, and the strategy we have been examining does preserve the immunity in question. On closer scrutiny, however, there seems nothing in the strategy which would really approve that initial idea. The immunity trivially follows from the fact that we have constructed the condition for some body part to belong to a subject in such a way that the immunity will be preserved. And the very concept of the subject is presupposed and explicitly brought into it. Indeed, even a Cartesian may find nothing objectionable about this condition. Thus this strategy does not seem to be a promising route for establishing the physical nature of the self.

\section{The Second and the Third Strategies}

The second and the third strategies, on the other hand, start by stipulating that, even under the abnormal circumstance, only $A$ is properly to be called 'my body'. We should remind ourselves of one of the objections to the first strategy, which claimed that my body should be a physically unified entity. The second and the 
third strategies subscribe to this suggestion, and make the assumption at the outset that the limit of my body should be the limit of A, even if I am apparently aware of some other body parts through bodily awareness. This is an assumption which we are not obliged to make, but which seems to be a reasonable one to make. Part of the justification for making it may be the thought that my body is an organism, and that physical unity is required by this idea.

Our target, on the other hand, is to show that $I$, the subject, am identical with the living human body $\mathrm{A}$, by appealing to considerations on immunity to error through misidentification relative to the owner. We still have some confidence in the observation that this immunity, if it obtains in judgements based on bodily awareness, may give substance to the idea that the subject is identical with the body of which it is aware. So, if the assumption that $\mathrm{A}$ is my body reasonably coheres with the immunity, then we will have a case for the desired conclusion. However, care must be taken. It matters how this immunity is accounted for. Our assessment of the first strategy has been that the immunity is indeed secured, but in such a way as to discredit the identification of the subject and the body. Therefore, our task is to show that the stipulation that $\mathrm{A}$ is my body consistently accommodates the immunity in an unproblematic manner that genuinely supports the desired conclusion.

The second strategy will be dealt with rather quickly, because it is not a real option for our purposes. It claims that in such cases as I am apparently aware of the condition of B's arm in addition to A, I have genuine bodily awareness of a body part which is not mine. This is how Cassam (1995: 319-328) has described the situation, although later Cassam (1997: 66) has abandoned this view in favour of the third strategy. That B's arm is not part of my body follows from the assumption that only $\mathrm{A}$ is my body, but this, on the current proposal, does not prevent me from having bodily awareness of it. Unfortunately, however, this is to give up the immunity to error relative to the owner. In our imagined situation, I may make a mistake of thinking that A's arm is bent while in fact B's arm is. When this happens, my judgement made on the basis of proprioceptive awareness is mistaken for the reason that I wrongly believe that the body part being bent is mine while in fact it is not.

On this conception, bodily awareness will lose the privilege of informing the subject exclusively of the condition of its own body, and it may as a result be seen as a form of perception parallel to sight. One knows the condition of body parts within the reach of one's bodily awareness, more or less in the way that one knows the colour and shape of objects within one's field of vision. Neither of them has a single entity as their sole object, and immunity to error will be relevant to neither. It may be quite reasonable to accept and develop this line of thought, but it will be at the cost of losing the ground for claiming that bodily awareness occupies any 
substantial role in a study of the self.

The third strategy starts with the same assumption as the second. Then it goes on to insist that, in the imagined case in which I am apparently aware of the condition of B's arm, we should not think that it really amounts to a genuine case of bodily awareness. It claims that the awareness I seem to have of B's body part should rather be seen as an illusion, or as some kind of clairvoyance. Having bodily awareness and only seeming to have it should sharply be distinguished. My body, which in our present proposal is assumed to be A, is the sole object of my bodily awareness properly so called. Martin (1995; 1997) and Cassam (1997) have pursued this approach, although Martin does not think that any consequence can be drawn on the nature of the self from these considerations. Thus, on this proposal, if I make any judgement on the basis of genuine bodily awareness, it is necessarily a judgement about the state of my body, A. It preserves immunity to error relative to the owner, by limiting bodily awareness only to awareness of the subject's own body. On the face of it, this explanation seems entirely satisfactory, and to provide us with a solution to our overall project.

However, there is a serious difficulty from which this strategy suffers. We have been supposing that I am almost always able to tell correctly the condition of B's arm through the apparent bodily awareness. We can also, if we like, tell science fiction stories of the causal mechanism underlying this capacity and its reliability. Given this, it will be unreasonable to claim that the apparent awareness I have of B's arm is mere illusion or clairvoyance. The current proposal must then make a compromise on this point and claim, rather, that it is a different form of awareness, which we may call ' $Q$ bodily awareness'. $Q$ bodily awareness is different from bodily awareness in that it informs the subject of conditions of body parts which are not its own.

It must readily be admitted from all sides that these two forms of awareness may be introspectively indiscriminable. As we mentioned earlier, under the imagined situation I may make such mistakes as thinking that A's arm is bent while in fact B's arm is, or I may be aware only that some arm is bent, and wonder whether it is A's or B's. If these cases are conceivable, it must be that bodily awareness and $\mathrm{Q}$ bodily awareness can be phenomenologically exactly similar. That is to say, the difference between them is not like the difference between sight and hearing, where it does not seem to make any sense to imagine a case in which one is aware of something either by sight or by hearing but one cannot tell which mode of awareness one is exercising. It should also be noted that $\mathrm{Q}$ bodily awareness is not illusion, and therefore the present point is not that some veridical awareness is indistinguishable form illusion. What we have here is two forms of awareness which may exhibit no phenomenologically detectable difference.

This raises a problem for the third strategy in the following way. The sugges- 
tion was that if my apparent bodily awareness is awareness of $\mathrm{A}$, then it is genuine bodily awareness and it does exhibit immunity to error relative to the owner. If it is awareness of some other body, then it is some other form of awareness. This in effect secures the immunity in cases of genuine bodily awareness only by introducing the possibility of another kind of error. That is, I may be in error in thinking of a particular case of awareness as bodily awareness, while in fact it is not. It may be a case of $Q$ bodily awareness. Therefore, the immunity obtains just in case I am fortunate enough not to have made the error of confusing bodily awareness with $Q$ bodily awareness.

This seems, as far as our project is concerned, to present us with a serious trouble. Clearly, the inquiry into the relationship between bodily awareness and the subject is motivated by the unquestioned assumption that bodily awareness is immediately accessible to the subject. That is, it is only in so far as it is thought that the subject has a very special direct access to the condition of a particular body that bodily awareness may seem to suggest anything of interest about the subject. The reason why immunity to error relative to the owner is thought to give substance to the idea that the subject is a corporeal entity is, as we have seen, that the body is so immediately presented to the subject that it cannot make a mistake in thinking of some body part to be its own, and that only identity between them has seemed capable of explaining the special connexion.

On the other hand, our current strategy characterises bodily awareness in such a way that the immunity will be preserved by compromising the immediacy we have of the form of awareness. This, however, is a concession we cannot afford. If we thus have to concede that, although the subject is indeed free from confusing its own body with some other body as far as the body part is presented to it through bodily awareness, mistake is still conceivable as to whether it is in fact through bodily awareness that the body part is presented, that is, it is possible to confuse bodily awareness with $\mathrm{Q}$ bodily awareness, then the distinctively close association between the subject and the body will be impaired after all. The current proposal has merely moved the point at which the special connexion can be disrupted from one place to another. Of course, pointing this out is not to claim that the view under consideration is incoherent. On the contrary, it is in itself reasonable and consistent. However, what we have seen is that, as in the case of the first and the second strategy, the third strategy has no force by itself to establish the physical nature of the self.

\section{Conclusion}

Let us look back how our argument proceeded. First we observed that in bodily awareness we are directly aware of a particular object, our own body. Then 
we went on to discuss the phenomenon of immunity to error through misidentification, and examined how immunity to error relative to the owner, if it obtained, would support the thesis that the subject of bodily awareness is identical with the object of that very awareness, and therefore that the subject of experience is a corporeal entity. Then we faced the problem case of apparently being aware of someone else's body, which would threaten the purported immunity, and went through each of three possible strategies that might be pursued. None of them, however, proved capable of supporting the thesis we intended to establish.

What is the moral to be drawn from all of this? Certainly, this is far from sufficient to demonstrate that it is wrong to think that the self is a corporeal entity. What we have seen is only that one possible route to that conclusion is not available. There might be other ways of reaching the conclusion, and in that sense our achievement is undoubtedly minimal. However, it is held by some philosophers that bodily awareness provides us with the strongest anti-Cartesian case. Then one might be led to think that to challenge the spell of Cartesianism is more demanding a task than it is believed to be. Moreover, Cartesianism is not the only position which might benefit from our observations. We should remind ourselves that we started with the assumption that there are such entities as subjects of experience, and that the first-personal pronoun ' $I$ ' used in judgements in which one ascribes experiences to oneself refers to oneself. One might think that we now have some reason to dispute this modest assumption. Indeed, one might have detected a slight sense of affinity that the view presented in this paper has to early Wittgenstein's remark in the Tractatus (5.632) that the subject does not belong to the world.

\section{References}

Anscombe, G.E.M. (1962), 'On Sensations of Position', Analysis, 22 : 55-58.

Bermúdez, J.L. (1998), The Paradox of Self-Consciousness (Cambridge, Mass. : MTT Press).

Bermúdez, J.L., Marcel, A., and Eilan, N. (eds.) (1995), The Body and the Self (Cambridge, Mass. : MIT Press).

Brewer, B. (1995), 'Bodily Awareness and the Self', in Bermúdez, Marcel, and Eilan (1995), 291-309.

Cassam, Q. (1995), 'Introspection and Bodily Self-Ascription', in Bermúdez, Marcel, and Eilan (1995), 311-336.

- (1997), Self and World (Oxford: Clarendon Press).

Evans, G. (1982), The Varieties of Reference, edited by J. McDowell (Oxford: Clarendon Press).

Martin, M. (1995), 'Bodily Awareness: A Sense of Ownership', in Bermúdez, Marcel, and Eilan (1995), 267-289.

- (1997), 'Self-Observation', European Journal of Philosophy, 5 : 119-140.

O'Shaughnessy, B. (1980), The Will (Cambridge: Cambridge University Press).

Shoemaker, S. (1968), 'Self-Reference and Self-Awareness', repr. in Shoemaker (1984), 6-18.

(1984), Identity, Cause, and Mind (Cambridge: Cambridge University Press). 
Wittgenstein, L. (1958), The Blue and Brown Books (Oxford: Basil Blackwell). (1961), Tractatus Logico-Philosophicus, trans. D.F. Pears and B.F. McGuinness (London: Routledge \& Kegan Paul). 